\title{
Hunting ghosts: the iconic stellar stream(s) around NGC 5907 under scrutiny ${ }^{\star}$
}

\author{
Oliver Müller ${ }^{1}$, Ana Vudragović ${ }^{2}$ and Michal Bílek ${ }^{1}$ \\ 1 Observatoire Astronomique de Strasbourg (ObAS), Universite de Strasbourg - CNRS, UMR 7550, Strasbourg, France \\ e-mail: oliver .muller@astro.unistra.fr \\ 2 Astronomical Observatory, Volgina 7, 11060 Belgrade, Serbia
}

Received 7 November 2019 / Accepted 27 November 2019

\begin{abstract}
Stellar streams are regarded as crucial objects for testing galaxy formation models because their morphology traces the underlying potentials and their occurrence tracks the assembly history of the galaxies. The existence of one of the most iconic stellar streams, the double loop around NGC 5907, has recently been questioned by new observations with the Dragonfly telescope. This new work only finds parts of the stream, even though a $1 \sigma$ surface brightness limit of $30.3 \mathrm{mag} \operatorname{arcsec}^{-2}$ in the $g$ band was reached. Using $7.2 \mathrm{~h}$ of luminance $L$-band imaging with the Milanković 1.4-m telescope, we have reobserved the putative double-loop part to confirm or reject this assessment. We do not find signs of the double loop, but see only a single knee-shaped stellar stream. Comparing our results to the data by the Dragonfly team, we find the same features. Our observations reach a $1 \sigma$ surface brightness limit of $29.7 \mathrm{mag} \mathrm{arcsec}^{-2}$ in the $g$ band. These findings emphasize the need for independent confirmation of detections of very low-surface brightness features.
\end{abstract}

Key words. galaxies: individual: NGC 5907 - galaxies: formation - techniques: image processing - galaxies: interactions galaxies: structure

\section{Introduction}

Over the past years, deep imaging of nearby galaxies has revealed a plethora of low-surface brightness features, such as dwarf galaxies, stellar streams, shells, and tidal debris. These features unlock crucial information for galaxy formation and are regarded as tests for the current cosmological model ( $\Lambda \mathrm{CDM})$, in which galaxies are formed in a hierarchical fashion by accretion of smaller galaxies (Bullock \& Johnston 2005, but see also Bílek et al. 2018, 2019). Some fossils of these ancient accretion events are expected to be detectable today; the number of these ghosts and their phase-space correlation is a strong test for the $\Lambda$ CDM model of galaxy formation (e.g., Ibata et al. 2002; Erkal et al. 2017; Bonaca \& Hogg 2018; Pearson et al. 2019).

It has been known for a long time that streams in the Local Group are wound around the Milky Way (e.g., Ibata et al. 1997; Belokurov et al. 2007) and the Andromeda galaxy (Martin et al. 2014; McConnachie et al. 2018), based on star count studies. With the Gaia mission, the number of streams detected around the Milky Way has exploded (Malhan et al. 2018; Ibata et al. 2019). Outside of our Local Group, the detection of resolved stellar streams is an almost impossible task with current instrumentation; the stream around NGC 891 (Mouhcine et al. 2010) at $10 \mathrm{Mpc}$ sets the record of what can be achieved today. At larger distances, only integrated-light observations are able to

\footnotetext{
* The raw science, dark, bias, and flat field frames are available at the CDS via anonymous ftp to cdsarc.u-strasbg. fr (130.79.128.5) or via http: //cdsarc.u-strasbg.fr/viz-bin/cat/J/A+A/632/
} L13 uncover these fossils. One of the most iconic streams discovered by integrated-light observations is found around NGC 5907 at $14 \mathrm{Mpc}$ (Shang et al. 1998), where Martínez-Delgado et al. (2008) uncovered a double-looped structure that was not visible in the Shang et al. (1998) data. Noteworthy, follow-ups of this stream with Subaru/Suprime Cam (Laine et al. 2016) detected only the parts that were already visible in Shang et al. (1998), but not the double loop. The observation by Martínez-Delgado et al. (2008) was made with a modest telescope of only 0.5-m diameter in size and was conducted by dedicated amateur astronomers. Since then, many more stellar streams around other giant galaxies have been detected by this amateur astronomer team, see, for instance, Martínez-Delgado et al. (2010).

The search for stellar streams is not only conducted by amateurs. Several professional teams have taken up the task to find streams and shells around nearby galaxies (e.g., Watkins et al. 2014, 2015; Duc et al. 2015; Bílek et al. 2016; Crnojević et al. 2016; van Dokkum et al. 2019). One of these teams, using the Dragonfly telescope (Danieli et al. 2019), has reobserved the iconic structure around NGC 5907 and to their surprise, found only part of the stream(s) reported by Martínez-Delgado et al. (2008), with no trace of the double loop (van Dokkum et al. 2019). Furthermore, they found a mismatch in the positions of the detected parts of the stream compared to those provided by Martínez-Delgado et al. (2008). This calls for an independent observation. To address this problem, we observed the putative double-loop part of NGC 5907 with the newly commissioned Milanković 1.4-m telescope (Samurović et al. 2018), located at the top of the Vidojevica mountain, Serbia. The results are presented in this Letter. 


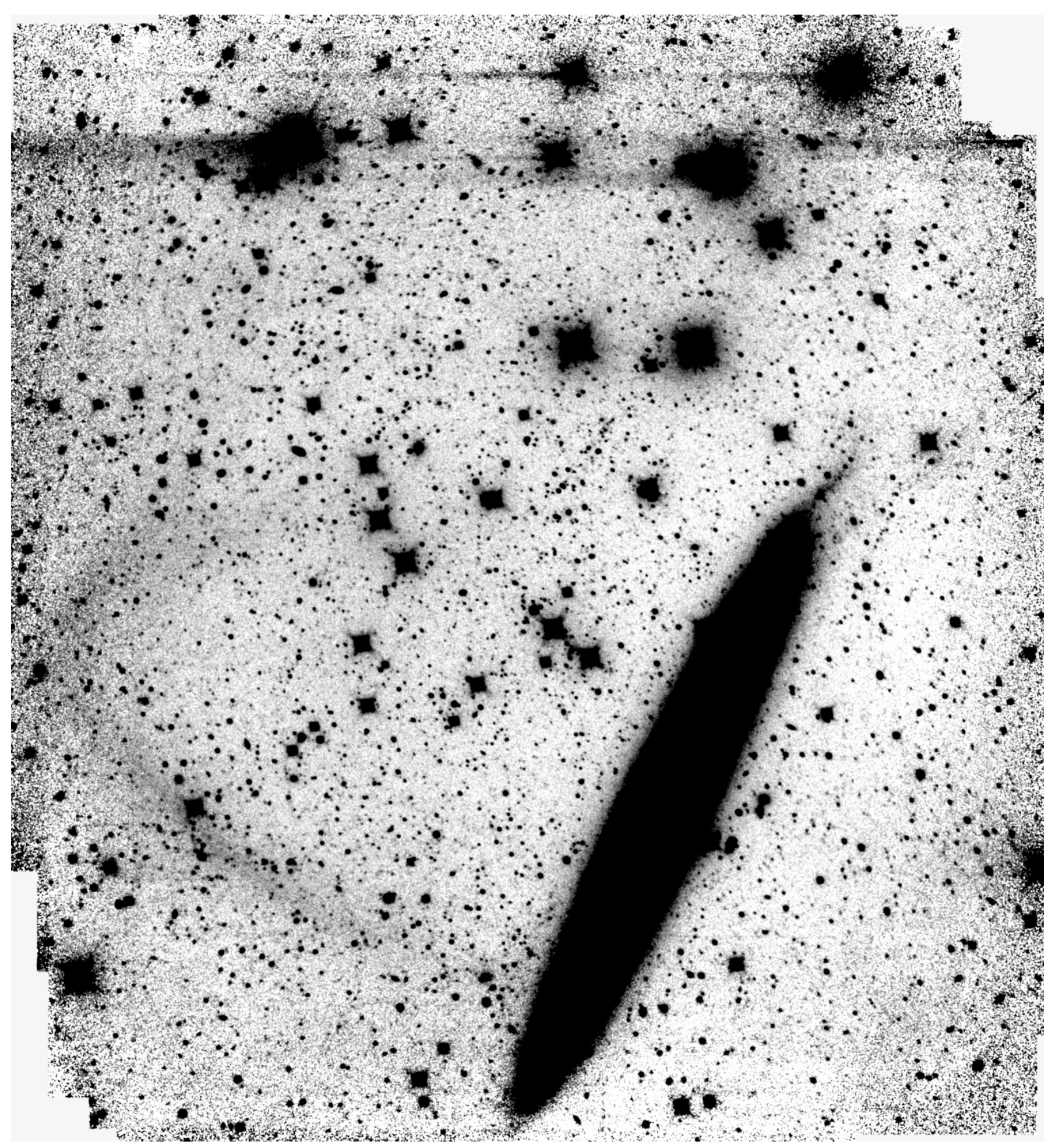

Fig. 1. Fully calibrated and stacked image of NGC 5907. The image was slightly enhanced with a Gaussian blur. The image is $18{ }^{\prime} .4 \times 20 \prime 2$ in size. North is to the top, and east is to the left.

\section{Observations and data reduction}

The galaxy NGC 5907 was observed on three consecutive nights (24-26 October 2019) with the 1.4-m Milanković telescope mounted at the Astronomical Station Vidojevica (Serbia), using an Andor IKONL CCD camera. The observations were taken at an airmass between $1.66<X<2.97$. The dark current is $0.02 \mathrm{e}^{-} \mathrm{s}^{-1}$. All images were taken in the luminance $L$-filter with 300-second exposures. The integrated exposure time reached was $7.2 \mathrm{~h}$. We had photometric nights with an average seeing of 1 ".4. The galaxy was at an altitude of $40^{\circ}$ at the beginning and went down to $20^{\circ}$ at the end of observations each night. The focal reducer delivers a square field-of-view with sides of 13!3. We applied a large dithering pattern randomized within a maximum offset of $4^{\prime}$, which is crucial when digging deep into the low-surface brightness regime at the 28-29 mag $\operatorname{arcsec}^{-2}$ level 
(Slater et al. 2009; Mihos 2019) and beyond (Trujillo \& Fliri 2016). This strategy provides a strong handle on the background subtraction, and on the removal of systematics arising from the instruments and observations, that is, from reflections or flatfielding artifacts.

The calibration of the images followed the standard procedure. A master bias frame was created from 10 single bias frames, using a median stack. A master dark frame was created from 10 single dark frames, again using a median stack. These master dark and bias frames were then subtracted from all individual flat fields and scientific exposures. A master flat field was made from 30 single twilight flat fields, which were normalized by their median value. The stack was again made with a median. The resulting master flat field was flat to the $1-2 \%$ level and was then applied to all individual dark-subtracted scientific exposures.

For the surface brightness limits we aimed to reach, it is crucial to carefully model the background. Approaches of background modeling, as is done in Source Extractor (Bertin \& Arnouts 1996), frequently also remove low-surface brightness features as part of the sky. Therefore, we took a custom approach. On every previously calibrated scientific exposure, we ran a source detection using Source Extractor with a $1 \sigma$ threshold, creating a segmentation map, which was then applied as a mask on the scientific exposures. Because Source Extractor will miss the faint outskirts of astronomical objects, we additionally dilated the mask with a $5 \sigma$ Gaussian kernel. The resulting masked image then only contained pixels corresponding to the background. The masked scientific exposures were then normalized by their median values, and stacked together. This stack will give a background model that is free from astronomical sources due to the large dithering pattern we applied. Because it is normalized, it can be scaled to the measured background values in the scientific exposures using the global background value estimated by Source Extractor and ultimately removed, resulting in our final fully calibrated and background-subtracted science frames. The final fully calibrated and dithering image reached a field of view of $18,8 \times 20 ! 3$. The python pipeline can be found on gitlab ${ }^{1}$.

The astrometry in the science frames was solved by astrometry.net (Lang et al. 2010). To create our final scientific image, we stacked all science frames with the IRAF imcombine command, using a median stack and an average sigma clipping rejection algorithm.

The zero-point of this stacked image was estimated using the Panoramic Survey Telescope And Rapid Response System (Pan-STARRS) DR2 star catalog (Magnier et al. 2016). We used DAOPHOT2 (Stetson 1987) aperture photometry to measure the instrumental $L$-band magnitudes of the stars. No $L$-band star catalogs are available so far, therefore we needed to transform the $L$ band into the more commonly used Sloan Digital Syk Survey (SDSS) bands. Javanmardi et al. (2016) successfully performed a linear transformation between the $L$ and the $r$ band and found only a minor dependence on the $g-r$ color (see their Fig. 1). Therefore, we calibrated our image to $g$-band standard stars using $g=c_{0} L+c_{1}$, based on 78 standard stars in a magnitude range between 17 and 22. The transformation is given with $c_{0}=1.03$ and $c_{1}=32.12 \mathrm{mag}$. The magnitude system of Pan-STARRS is in AB.

For the parts of the image with $100 \%$ coverage, the surface brightness limit was measured from the standard deviation in $10 \times 10 \operatorname{arcsec}^{2}$ boxes of empty pixels, meaning that they did not

\footnotetext{
https://gitlab.com/VoltarCH/milankovic-telescopereduction-pipeline
}

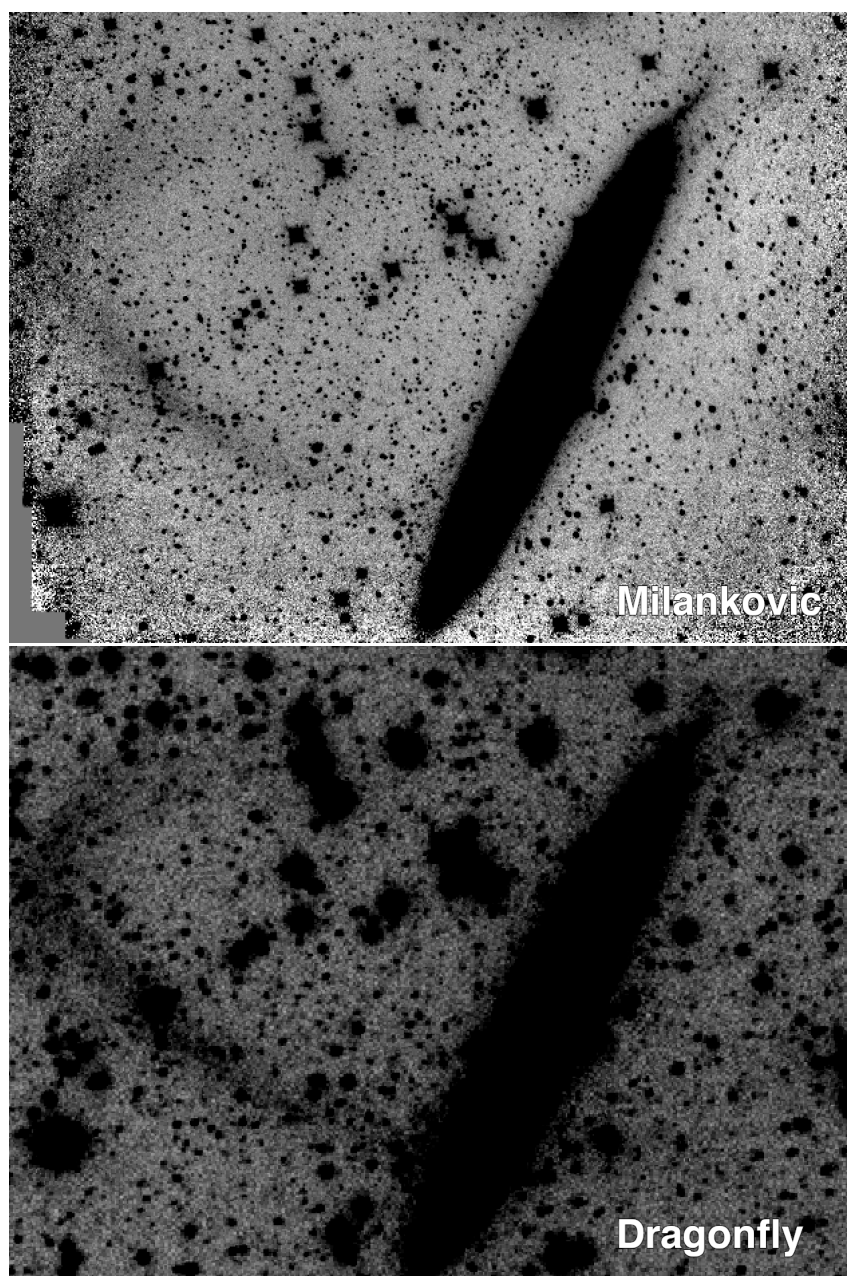

Fig. 2. Comparison between our observations (top) and the relevant part of the Dragonfly image (bottom), kindly provided by the Dragonfly team.

contain any (visible) stars. We estimate a $1 \sigma$ surface brightness limit of $29.7 \pm 0.04 \mathrm{mag} \operatorname{arcsec}^{-2}$ in the $g$ band (corresponding to a $3 \sigma$ limit of $28.4 \pm 0.04 \mathrm{mag} \operatorname{arcsec}^{-2}$ ). The error was estimated by the standard deviation of the measured surface brightness of 14 such boxes.

\section{Low surface brightness features}

In Fig. 1 we present the fully stacked and calibrated image of the surroundings of NGC 5907, slightly enhanced with a Gaussian blur. What low-surface brightness features does it reveal? We detect a narrow low-surface brightness feature stretching 10'.5 $(\approx 43 \mathrm{kpc}$ at $14 \mathrm{Mpc})$ from the disk of the galaxy toward the east, it then bends $4.2(\approx 17 \mathrm{kpc}$ at $14 \mathrm{Mpc})$ toward the north, where it gradually disappears. This morphology is reminiscent of stellar streams found in other galaxy groups, for example, around NGC 1052 (Müller et al. 2019).

In Fig. 2 we compare the relevant part of our image with the Dragonfly $g$-band image taken by van Dokkum et al. (2019). The shapes of the stream detected by us and by van Dokkum et al. (2019) are identical, which is also apparent when the two images are blinked. The question now is where the double loop is located. In Fig. 3 we indicate the contours of the double stream reported by Martínez-Delgado et al. (2008). There are no indications of these structures in our image. We applied the same 


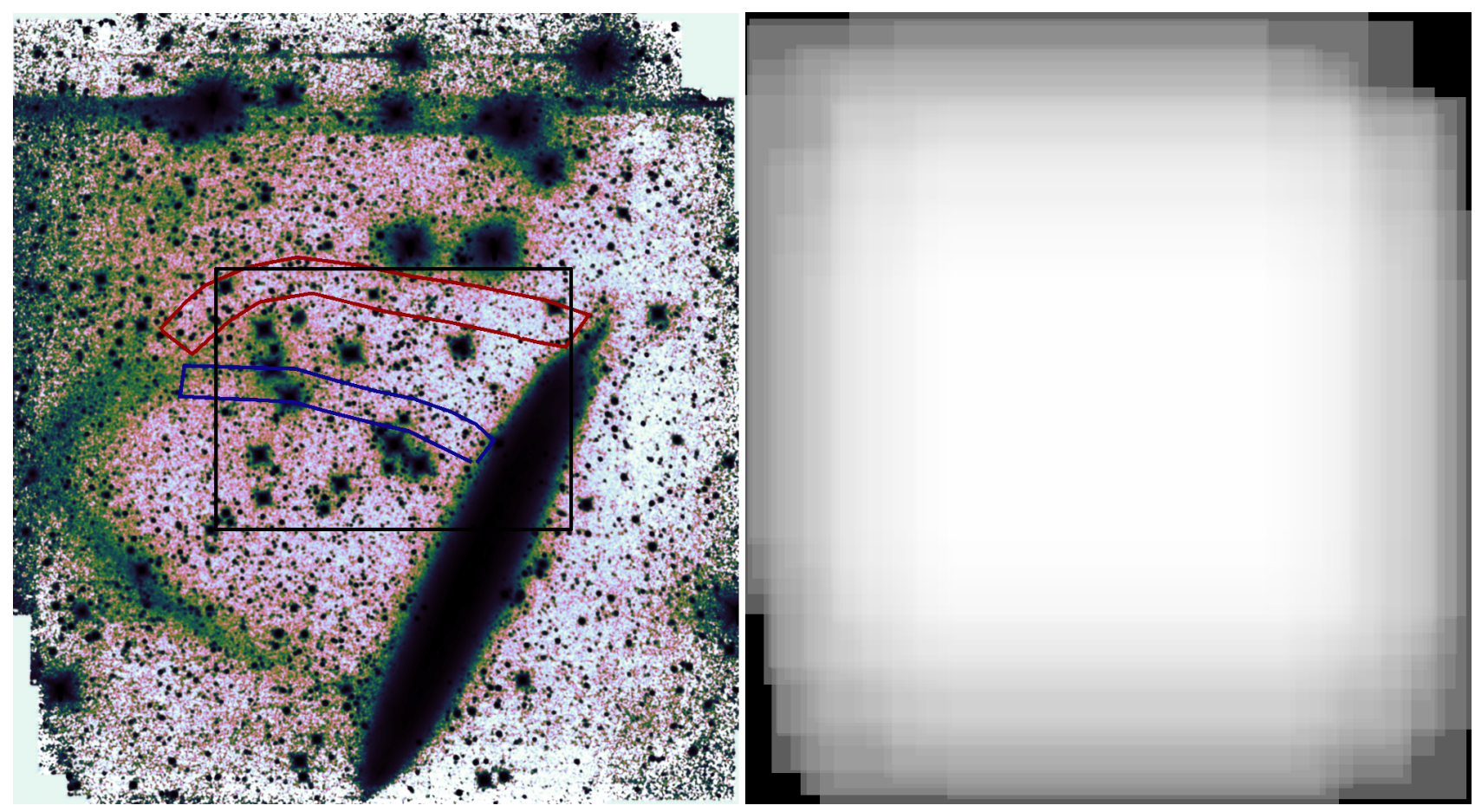

Fig. 3. Left: image enhanced with a Gaussian blur and a histogram equalization, as discussed in Martínez-Delgado et al. (2008). The paths indicate the positions of the missing parts of the streams east of the galaxy; this is the iconic part of the double loop system. The black box corresponds to the region where a 100\% coverage has been achieved. Right: weight map of the stack in a logarithmic representation. White corresponds to a $100 \%$ coverage, i.e., the area with 7.2-h exposure in total, black to $0 \%$.

post-processing techniques as Martínez-Delgado et al. (2008): a histogram equalization and a Gaussian blur. In these regions, we measure a $1 \sigma$ surface brightness limit of $29.7 \mathrm{mag} \mathrm{arcsec}^{-2}$ in the $g$ band (i.e. a $3 \sigma$ limit of $28.4 \mathrm{mag} \mathrm{arcsec}^{-2}$ ). However, we note that this was measured before the image was enhanced, giving a lower limit of what is detectable. In Fig. 3 we also show the part of the image with a $100 \%$ coverage of exposures, corresponding to the deepest part of the full stack. The two putative streams are located well within this area, therefore incompleteness of the data should play no role. In contrast, the part of the stream to the west (i.e., bottom right) of NGC 5907 cannot be detected in our image, butis visible in the Dragonfly images (see Fig. 2). This part coincides with an incomplete area of the image; see also our weight map in Fig. 3.

The faintest stars detected in our images are of about 25.8$26.0 \mathrm{mag}$ in the $g$ band, which is several magnitudes deeper than the detection limit of the SDSS, for instance. The magnitudes were estimated with simple aperture photometry on several hand-picked faint stars.

\section{Discussion and conclusion}

The recent paper by van Dokkum et al. (2019) has stirred a controversy that has been hotly debated at conferences and in the social media. To shed light on the discussion, we have independently taken deep data using a 1.4-m telescope to assess whether there is a double-loop structure, as found by Martínez-Delgado et al. (2008), or rather a simple knee-shaped stream, as found by van Dokkum et al. (2019). Our imaging is consistent with the latter, with no signs of a double loop.

We cannot assess whether there was a problem with the data reduction by Martínez-Delgado et al. (2008) because the data are unfortunately not available. Therefore, we can only rely on re-observations of the system. An argument raised in favor of the existence of a double loop is the fact that many amateur astronomers have repeatedly detected it. However, the data-reduction procedures adopted for the amateur images are not always transparent. The fact that now two completely independent professional teams were unable to confirm the double loop sheds some doubts on at least some of the previously found very low surface brightness features. The limiting surface brightness of amateur images is rather difficult to assess. Both our data and the data by van Dokkum et al. (2019) have applied large dithering patterns, which indicates that the handling of the data could be one of the culprits causing the disagreement. In this respect, it is also striking that in the deep Subaru/Suprime Cam data by Laine et al. (2016) no double-loop feature was visible, even though it was not discussed in their work. It seems that professionally handled data always yield a single stream, while data processed by amateur astronomers uncover more features ${ }^{2}$. As a benchmark test, the faintest stars in the different images could be compared. In our images, we reach faint stars up to $g=26.0 \mathrm{mag}$.

Furthermore, the exposure time is puzzling. For example, the reobservation of NGC 5907 by the astro-photographer Stefan Binnewies, using a 0.6-m telescope and $24 \times 600$ s exposures, that is, $4 \mathrm{~h}$ in total in the $L$ band, clearly shows part of the double loop. It is highly unlikely that our $86 \times 300 \mathrm{~s}$ exposures, that is, $7.2 \mathrm{~h}$ in total in the same band, is unable to detect the double loop while having a larger collecting area of more than a factor of $5^{3}$. Even more surprising is that the brighter parts of the

\footnotetext{
2 Even though not all amateur astronomers recover the two distinct features of the double loop.

3 However, a comparison between the exposure times of different telescopes is strictly speaking only valid if the CCD sensitivity, the pixel
} size, and the observation site are taken into account. 
missing double loop appear as bright in the astro-photographer's images $^{4}$ as the parts of the stream we detected here. If the apparent surface brightnesses are similar, then we should be able to detect both features as well, which we apparently do not. This again indicates a problem: the brightness in their post-processing is not preserved.

After discussing all this, the question is whether there still is a way to reconcile all observations. One difference between professional and amateur systems might be the spectral sensitivities of the CCDs and the transmission curves of the filters. In this case, different features would appear more or less prominent, for example, if the emission comes from [OII] at $372.7 \mathrm{~nm}$, which is just outside the typical SDSS $g$-band transmission curve. However, this would mean that by digging deeper or changing the filters, for instance, by employing the SDSS $u$ band, we should eventually be able to see the double loop if it is there.

To summarize this Letter, we have acquired deep, highquality data with a 1.4-m telescope, reaching a depth of $29.7 \mathrm{mag} \mathrm{arcsec}^{-2}$ in the $g$ band, and find no signs of the iconic double loop around NGC 5907. Instead, we find a single kneeshaped stream, as was independently found by the Dragonfly team. These results emphasize the need for an independent confirmation of the reliability of the detected low-surface brightness features by using different telescopes, dedicated observing strategies, and consistent scientific processing techniques.

This work used following scientific software: Astrometry.net (Lang et al. 2010), the DAOPHOT2 suite (Stetson 1987), and IRAF (Tody 1993); as well as following python3 packages: Astropy (Astropy Collaboration 2013), ccdproc (Craig et al. 2015), and sep (Barbary 2016), which is a python implementation of Source Extractor (Bertin \& Arnouts 1996).

Acknowledgements. We thank the referee for the constructive report, which helped to clarify and improve the manuscript. O. M. is grateful to the Swiss National Science Foundation for financial support. The authors thank Pieter van Dokkum for fruitful discussions about the observations and providing the Dragonfly data shown in Fig. 2. The authors also thank Marina Rejkuba and Rodrigo Ibata for valuable comments. This work was supported by the Ministry of Education, Science and Technological Development of the Republic of Serbia (MESTDRS) through project no. 176021, "Visible and Invisible Matter in Nearby Galaxies: Theory and Observations". We acknowledge the financia support by the European Commission through project BELISSIMA (BELgrade Initiative for Space Science, Instrumentation and Modelling in Astrophysics, call FP7-REGPOT-2010-5, contract No. 256772), which was used to procure the Milanković $1.4 \mathrm{~m}$ telescope with the support from the MESTDRS. We thank the technical operators at the Astronomical Station Vidojevica (ASV), Miodrag Sekulić and Petar Kostić for their excellent work.

\section{References}

Astropy Collaboration (Robitaille, T. P., et al.) 2013, A\&A, 558, A33 Barbary, K. 2016, J. Open Source Softw., 1, 58

Belokurov, V., Evans, N. W., Irwin, M. J., et al. 2007, ApJ, 658, 337 Bertin, E., \& Arnouts, S. 1996, A\&AS, 117, 393

Bílek, M., Cuillandre, J. C., Gwyn, S., et al. 2016, A\&A, 588, A77

Bílek, M., Thies, I., Kroupa, P., \& Famaey, B. 2018, A\&A, 614, A59

Bílek, M., Thies, I., Kroupa, P., \& Famaey, B. 2019, 355 IAU Symp., in press [arXiv:1908.07537]

Bonaca, A., \& Hogg, D. W. 2018, ApJ, 867, 101

Bullock, J. S., \& Johnston, K. V. 2005, ApJ, 635, 931

Craig, M. W., Crawford, S. M., Deil, C., et al. 2015, Astrophysics Source Code Library [record ascl:1510.007]

Crnojević, D., Sand, D. J., Spekkens, K., et al. 2016, ApJ, 823, 19

Danieli, S., Lokhorst, D., Zhang, J., et al. 2019, ApJ, submitted [arXiv:1910.14045]

Duc, P.-A., Cuillandre, J.-C., Karabal, E., et al. 2015, MNRAS, 446, 120

Erkal, D., Koposov, S. E., \& Belokurov, V. 2017, MNRAS, 470, 60

Ibata, R. A., Wyse, R. F. G., Gilmore, G., Irwin, M. J., \& Suntzeff, N. B. 1997, AJ, 113, 634

Ibata, R. A., Lewis, G. F., Irwin, M. J., \& Quinn, T. 2002, MNRAS, 332, 915

Ibata, R. A., Malhan, K., \& Martin, N. F. 2019, ApJ, 872, 152

Javanmardi, B., Martinez-Delgado, D., Kroupa, P., et al. 2016, A\&A, 588, A89

Laine, S., Grillmair, C. J., Capak, P., et al. 2016, AJ, 152, 72

Lang, D., Hogg, D. W., Mierle, K., Blanton, M., \& Roweis, S. 2010, AJ, 139, 1782

Magnier, E. A., Schlafly, E. F., Finkbeiner, D. P., et al. 2016, ArXiv e-prints [arXiv:1612.05242]

Malhan, K., Ibata, R. A., \& Martin, N. F. 2018, MNRAS, 481, 3442

Martin, N. F., Ibata, R. A., Rich, R. M., et al. 2014, ApJ, 787, 19

Martínez-Delgado, D., Peñarrubia, J., Gabany, R. J., et al. 2008, ApJ, 689, 184

Martínez-Delgado, D., Gabany, R. J., Crawford, K., et al. 2010, AJ, 140, 962

McConnachie, A. W., Ibata, R., Martin, N., et al. 2018, ApJ, 868, 55

Mihos, J. C. 2019, ArXiv e-prints [arXiv:1909. 09456]

Mouhcine, M., Ibata, R., \& Rejkuba, M. 2010, ApJ, 714, L12

Müller, O., Rich, R. M., Román, J., et al. 2019, A\&A, 624, L6

Pearson, S., Starkenburg, T. K., Johnston, K. V., et al. 2019, ApJ, 883, 87

Samurović, S., Djurasević, G., Cvetković, Z., Pavlović, R., \& Vince, O. 2018, Publications de l'Observatoire Astronomique de Beograd, 98, 333

Shang, Z., Zheng, Z., Brinks, E., et al. 1998, ApJ, 504, L23

Slater, C. T., Harding, P., \& Mihos, J. C. 2009, PASP, 121, 1267

Stetson, P. B. 1987, PASP, 99, 191

Tody, D. 1993, in Astronomical Data Analysis Software and Systems II, eds. R. J. Hanisch, R. J. V. Brissenden, \& J. Barnes, ASP Conf. Ser., 52, 173

Trujillo, I., \& Fliri, J. 2016, ApJ, 823, 123

van Dokkum, P., Gilhuly, C., Bonaca, A., et al. 2019, ApJ, 883, L32

Watkins, A. E., Mihos, J. C., Harding, P., \& Feldmeier, J. J. 2014, ApJ, 791, 38

Watkins, A. E., Mihos, J. C., \& Harding, P. 2015, ApJ, 800, L3

\footnotetext{
4 See their stacked and enhanced image on http:// www . capella-observatory.com/ImageHTMLs/Galaxies/ NGC5907Prim.htm
} 